\title{
ANDES

www.scielo.cl

\section{Significado y participación social del hombre al transformarse en padre por primera vez}

\author{
Meaning and social participation of man when become a father for the first time
}

\author{
Francisca Márquez-Doren ${ }^{\mathrm{a}}$, Camila Lucchini-Raies ${ }^{\mathrm{a}}$, María Rita Bertolozzi ${ }^{\mathrm{b}}$
}

aEnfermera-Matrona. Escuela de Enfermería, Pontificia Universidad Católica de Chile. Santiago, Chile

bEnfermera-Matrona, Escola de Enfermagem, Universidade de São Paulo. São Paulo, Brasil

Recibido: 18 de febrero de 2020; Aceptado: 17 de septiembre de 2020

¿Qué se sabe del tema que trata este estudio?

La paternidad ha cambiado hacia un mayor involucramiento en el cuidado de los hijos/as. Cuando el padre se involucra en la crianza se fortalece el sistema familiar, lo que contribuye al desarrollo infantil saludable y al vínculo afectivo entre ellos.
¿Qué aporta este estudio a lo ya conocido?

Comprender la experiencia de paternidad desde la perspectiva del propio padre y su pareja, y al niño/a como protagonista en el desarrollo de la vinculación con el padre. Surge como oportunidad para el sistema de salud conocer las necesidades de los padres.

\section{Resumen}

Objetivo: Analizar los significados atribuidos a la paternidad por hombres al ser padres por primera vez. Participantes y Método: Investigación cualitativa de diseño Hermenéutico-Dialéctico. La recolección de datos fue realizada a través de entrevistas en profundidad a padres y madres (10 de cada uno), de niños/as mayores de 12 meses. El análisis de la información se llevó a cabo a través del análisis de discurso de los participantes y su articulación con el contexto histórico-social y los referenciales teóricos de la investigación. Resultados: El significado y participación social del hombre al transformarse en padre por primera vez se conformó por cinco categorías: significado de la experiencia de la paternidad; significado de la paternidad desde una perspectiva de género; significado de la experiencia en la relación padre-hijo/a; significado de la experiencia de paternidad en la relación de pareja; y ejercitando la paternidad y su relación con el sistema de salud. Conclusiones: Se requiere implementar estrategias de inclusión del padre en la crianza y cuidado de los hijos/as, favoreciendo su participación, y de este modo, el crecimiento y desarrollo integral del niño/a concebido en los diversos tipos de familia.
Palabras clave: Enfermería en Salud Comunitaria; Paternidad; Cuidado del Niño; Hermenéutica

Correspondencia:

Camila Lucchini-Raies

clucchin@uc.cl 


\begin{abstract}
Objective: To analyze the meanings attributed to fatherhood by first-time fathers. Participants and Method: Qualitative research of Hermeneutic-Dialectic design. Data collection was carried out through in-depth interviews with fathers and mothers (10 of each) of children older than 12 months. The analysis of the data was carried out through the participants' discourse analysis and their articulation with the historical-social context and the theoretical references of the research. Results: The meaning and social participation of men when they became a first-time father were grouped in five categories: meaning of the fatherhood experience; meaning of fatherhood from a gender perspective; meaning of the experience in the father-child relationship; meaning of the parenting experience in the couple relationship; and exercising fatherhood and its relationship with the health system. Conclusions: It is necessary to implement strategies to include the father in the upbringing and care of the children, favoring his participation, and thus, the growth and comprehensive development of the child conceived in the different types of families.
\end{abstract}

Keywords:

Community Health

Nursing;

Paternity;

Child Care;

Hermeneutics

\section{Introducción}

La composición de la familia y los roles ejercidos dentro de ella han variado a lo largo del tiempo tanto nacional como internacionalmente. Asimismo, el ejercicio de la paternidad ha experimentado grandes cambios en el transcurso de la historia, siendo casi inexistente en los inicios de la sociedad chilena, tomando un rol más participativo a comienzos de la década de los noventa, donde el hombre empieza a tener un rol más protagónico en algunos escenarios de crianza y a ser considerado en políticas de infancia ${ }^{1,2}$.

Es así como, el año 2006 se crea el "Sistema de Protección Integral a la Primera Infancia, Chile Crece Contigo", con el propósito de fortalecer el desarrollo infantil saludable como determinante primordial en la salud de las futuras generaciones. Este sistema contempla la incorporación de la estimulación prenatal saludable para el niño/a y su familia, incentivando la participación del padre desde la gestación, como forma de involucrarlo en la crianza de su hijo/a $\mathrm{a}^{3,4}$.

Se ha descrito que la inclusión de los padres en la crianza fortalece el sistema familiar, contribuyendo al desarrollo óptimo del niño/a, sus padres y familia y el establecimiento del vínculo afectivo entre ellos. Lo anterior tendría un efecto protector frente a la violencia y los trastornos afectivos ${ }^{5,6}$. Sin embargo, los estudios señalan que el grado de involucramiento del padre en la crianza es escaso y cuando participa, ejerce solo el rol de acompañamiento y apoyo de la madre ${ }^{7-10}$.

Los resultados anteriores dan cuenta de los efectos que tiene que los padres se involucren en la crianza de sus hijos/as, sin embargo, no especifican cuál es la participación social del hombre al transformarse en padre ni el contexto político-social en que se da el ejercicio de la paternidad. La participación social se entiende como las formas en que las personas se insertan en la socie- dad, las cuales repercuten sobre las manifestaciones de salud y de enfermedad, considerando a la persona como ser histórico, determinado por el espacio y época en la que vive ${ }^{11}$.

La participación social del hombre al transformarse en padre es un conocimiento que no ha sido develado en el contexto social ni dentro del sistema de salud chileno. Aun cuando se ha establecido una política de desarrollo integral de infancia, que considera la participación paterna, no se han evaluado sus efectos desde la perspectiva de los propios padres ${ }^{12-14}$. Considerando las brechas existentes en el conocimiento sobre lo que experimentan los hombres al transformarse en padres, se planteó como objetivo interpretar los significados atribuidos a la experiencia de transformarse en padre por primera vez desde la perspectiva de los hombres y sus parejas en el contexto de la sociedad chilena.

\section{Participantes y Método}

Estudio cualitativo de diseño hermenéutico dialéctico, que busca interpretar el relato en tiempo presente de la vivencia humana, establecer correlaciones entre lo ocurrido y el contexto histórico-social en el que se desenvuelve su vida ${ }^{15}$. El reporte de la metodología se cauteló aplicando el instrumento COREQ (Consolidated Criteria for Reporting Qualitative Research) ${ }^{16}$.

Los participantes de este estudio fueron hombres viviendo la experiencia de ser padres por primera vez y sus parejas, pertenecientes a los cinco quintiles de ingreso, usuarios de centros de salud públicos y privados de la Región Metropolitana de Santiago. Los criterios de inclusión fueron: ser hombres, mayores de 18 años, con hijos/as sanos mayores de un año. Además, se entrevistó a sus parejas también mayores de edad. Por tanto, se seleccionaron hombres y sus pare- 
jas, que cumplieron los criterios de inclusión a través del muestreo intencionado de bola de nieve. Quienes accedieron a participar fueron contactados telefónicamente por la investigadora principal (IP) para acordar el lugar y hora de la entrevista según su conveniencia.

La técnica de recolección de información fue la entrevista en profundidad y la aplicación de un cuestionario de caracterización sociodemográfica. Las entrevistas fueron realizadas por la IP y una co-investigadora, grabadas con previo consentimiento y transcritas verbatim. La pregunta que guio la entrevista de hombres fue: ¿Qué ha significado para usted convertirse en padre? y de las parejas fue: ¿Qué ha significado, desde su perspectiva, el convertirse en padre para su pareja?

El criterio para determinar el número de participantes fue la saturación de la información ${ }^{17}$, alcanzada cuando los datos comenzaron a repetirse, lo que ocurrió en las entrevistas de la décima pareja. Se invitaron a 13 parejas de las cuales dos rechazaron la invitación. Se realizaron 20 entrevistas ( 10 hombres y 10 mujeres) entre mayo y septiembre de 2014. De las parejas entrevistadas, en dos casos participó un solo miembro, dado que no fue posible acordar entrevistas con ambos. De manera de anonimizar los relatos de los participantes, a cada entrevista se le asignó una sigla utilizando la letra $\mathrm{H}$ para los testimonios de hombres y la letra $\mathrm{M}$ para los testimonios de mujeres, un número con la frase extraída y otro con el quintil de ingreso. Ejemplo, H4/15/3 significa que el relato pertenece al hombre cuatro, frase temática 15, quintil de ingreso 3.

Para el análisis de la información se utilizó el método hermenéutico dialéctico ${ }^{(15)}$, que considera a los participantes como actores sociales situados en su contexto específico. Incluye dos niveles de análisis interpretativo: 1) valoración del contexto social y perspectiva histórica y 2) análisis de los testimonios y su articulación con el contexto histórico-social y los refe- renciales teóricos de la investigación ${ }^{15}$. En este artículo se llevó a cabo el segundo nivel interpretativo, dado que el primero fue realizado en una etapa previa ${ }^{1,8}$. Los niveles de análisis interpretativo y sus etapas se detallan en la tabla 1.

Esta investigación fue aprobada por los comités de ética correspondientes (Resoluciones: 24102013 y 112013).

\section{Resultados}

Los participantes del estudio fueron 10 hombres y 10 mujeres entre 21 y 43 años. Todos los participantes tenían enseñanza media completa o educación superior, existiendo una relación directa entre el nivel de escolaridad y el quintil de ingreso al que pertenecían. Dieciocho de los 20 participantes se encontraban casados o viviendo en pareja y con hijos/as entre 12 a 48 meses de edad (tabla 2).

El significado y participación social del hombre al transformarse en padre por primera vez se conformó por cinco categorías (tabla 3), cuatro de las cuales fueron establecidas previamente en base a la revisión de la literatura y una quinta que emergió durante la fase de análisis. Cada categoría se constituyó por subcategorías, las que se compusieron de frases temáticas construidas a partir del análisis de los relatos de los participantes.

\section{Categoría 1: Significado de la experiencia de la paternidad}

La paternidad por primera vez mostró que los hombres experimentan una emoción intensa, que los colma y en cierto sentido los sobrepasa en términos de lo que habían proyectado. Están ciertos que es un cambio dinámico, enfrentando todos los días algo nuevo:

Tabla 1. Niveles de análisis interpretativo del método hermenéutico dialéctico y sus etapas

\begin{tabular}{ll}
\hline Nivel & Etapa \\
\hline $\begin{array}{l}\text { Primer nivel: valoración del contexto } \\
\text { social y perspectiva histórica }\end{array}$ & $\begin{array}{l}\text { 1. Descripción de coyuntura socio-económica/política que incluye el contexto histórico, las condi- } \\
\text { ciones específicas de vida/trabajo, las políticas existentes en relación a la temática y la perspectiva } \\
\text { de género. Esta etapa se llevó a cabo a través de un análisis documental' }\end{array}$ \\
& $\begin{array}{l}\text { 2. Establecimiento de categorías generales para el abordaje de las entrevistas: } \\
\text { a) significado sobre paternidad }\end{array}$ \\
& $\begin{array}{l}\text { b) significado sobre el desarrollo afectivo del niño/a con su padre } \\
\text { c) significado sobre la paternidad desde una perspectiva de género }\end{array}$ \\
$\begin{array}{ll}\text { Segundo nivel: análisis de los } & \text { 1. Ordenamiento de los datos a través de un mapeo de la información recogida durante el trabajo } \\
\text { testimonios y su articulación } & \text { de campo } \\
\text { con el contexto histórico-social } & \text { 2. Clasificación de los datos mediante una lectura exhaustiva y repetida de los textos, para la ela- } \\
\text { y los referenciales teóricos de la } & \text { boración de frases temáticas a través de la técnica de análisis de discurso } \\
\text { investigación } & \text { 3. Análisis final para la articulación entre los datos y los referenciales teóricos }\end{array}$ \\
\hline
\end{tabular}


Tabla 2. Características sociodemográficas de los participantes

\begin{tabular}{|c|c|c|c|c|c|c|c|}
\hline Parejas & $\begin{array}{l}\text { Identificación con } \\
\text { nombre ficticio }\end{array}$ & Edad & $\begin{array}{l}\text { Quintil de } \\
\text { ingresos }\end{array}$ & Escolaridad & Estado civil & $\begin{array}{l}\text { Sexo del } \\
\text { hijo/a }\end{array}$ & $\begin{array}{c}\text { Edad del hijo/a } \\
\text { en meses }\end{array}$ \\
\hline \multirow[t]{2}{*}{ P1 } & $J P$ & 32 & 5 & Nivel de Magíster & Casados & $\mathrm{F}$ & 22 \\
\hline & Trinidad & 32 & 5 & Nivel de Magíster & & & \\
\hline \multirow[t]{2}{*}{ P2 } & Andrés & 43 & 5 & Nivel de Magíster & Casados & $\mathrm{F}$ & 17 \\
\hline & Manuela & 40 & 5 & Universitaria completa & & & \\
\hline P3 & Alejandra & 21 & 1 & Técnico incompleto & Sin vínculo & $\mathrm{F}$ & 15 \\
\hline \multirow[t]{2}{*}{ P4 } & Alberto & 26 & 4 & Universitaria completa & Conviven & $\mathrm{F}$ & 15 \\
\hline & Andrea & 26 & 4 & Universitaria incompleta & & & \\
\hline P5 & Eduardo & 31 & 3 & Técnico completo & Sin vínculo & $\mathrm{F}$ & 18 \\
\hline \multirow[t]{2}{*}{ P6 } & Opinión de padre & 28 & 4 & Enseñanza media completa & Conviven & $\mathrm{F}$ & 48 \\
\hline & Estefany & 29 & 4 & Técnico incompleto & & & \\
\hline \multirow[t]{2}{*}{ P7 } & Roberto & 32 & 2 & Enseñanza media completa & Conviven & $\mathrm{F}$ & 19 \\
\hline & Beyonce & 33 & 2 & Media completa & & & \\
\hline \multirow[t]{2}{*}{ P8 } & Mauricio & 34 & 3 & Media completa & Casados & M & 18 \\
\hline & Baby & 32 & 3 & Media completa & & & \\
\hline \multirow[t]{2}{*}{ P9 } & Atahualpa & 26 & 4 & Universitaria incompleta & Conviven & $M$ & 12 \\
\hline & Ana & 23 & 4 & Técnica completa & & & \\
\hline \multirow[t]{2}{*}{$\mathrm{P} 10$} & David & 30 & 4 & Media completa & Conviven & $\mathrm{F}$ & 20 \\
\hline & Camila & 30 & 4 & Técnica completa & & & \\
\hline \multirow[t]{2}{*}{ P11 } & Francisco & 37 & 5 & Universitaria completa & Casados & M & 48 \\
\hline & Andrea & 37 & 5 & Universitaria completa & & & \\
\hline
\end{tabular}

Tabla 3. Categorías y subcategorías de análisis

\begin{tabular}{|c|c|}
\hline Categorías & Subcategorías \\
\hline Significado de la experiencia de la paternidad & $\begin{array}{l}\text { - Emoción intensa } \\
\text { - Desarrollo de un amor profundo } \\
\text { - Cambio de prioridades } \\
\text { - Experiencia de los padres respecto de la relación con su propio padre }\end{array}$ \\
\hline $\begin{array}{l}\text { Significado de la paternidad desde una perspectiva } \\
\text { de género }\end{array}$ & $\begin{array}{l}\text { - La experiencia del hombre que es padre frente a un rol asociado a lo femenino } \\
\text { - Responsabilidad frente al rol de proveedor y formador }\end{array}$ \\
\hline $\begin{array}{l}\text { Significado de la experiencia en la relación } \\
\text { padre-hijo/a }\end{array}$ & $\begin{array}{l}\text { - Sensación del padre de ser prescindible, desconexión inicial } \\
\text { - Proceso de desarrollo de un vínculo con su hijo/a, conexión } \\
\text { - Consolidando la relación con su hijo/a }\end{array}$ \\
\hline $\begin{array}{l}\text { Significado de la experiencia de paternidad en la } \\
\text { relación de pareja }\end{array}$ & $\begin{array}{l}\text { - La decisión de ser padre/madre se comparte en pareja } \\
\text { - Periodo de adaptación y cambios en la relación de pareja } \\
\text { - Red de apoyo de la familia extendida } \\
\text { - Estatus de ayuda mutua y apoyo en la relación de pareja }\end{array}$ \\
\hline $\begin{array}{l}\text { Ejercitando la paternidad y su relación con el sistema } \\
\text { de salud }\end{array}$ & $\begin{array}{l}\text { - Consideración del padre en el proceso de crianza de los niños/as } \\
\text { - Necesidades de los padres no siempre atendidas } \\
\text { - Exclusión de los padres del proceso de crianza de los niños/as } \\
\text { - Formas de incluir a los padres }\end{array}$ \\
\hline
\end{tabular}


"No es tan solo una experiencia diferente...por ser experimentada por primera vez...Es la experiencia que lo diferencia todo" (H5/2/3).

También, los padres requieren adecuarse a una situación nueva que marca sus vidas. Viviéndola como un cambio positivo pero radical, que implica modificaciones profundas, además, de dejar de lado actividades que antes realizaban periódicamente, incluso ejemplificado como "dejar de ser un adolescente para ser un adulto" (H6/5/4).

"El padre siente responsabilidad por su nueva familia...retoma sus estudios para darle un futuro mejor...a su hija y su pareja...él es capaz de soportar cosas no tan gratas por su hija” (M3/19/1).

Vivir la experiencia de paternidad evoca la propia experiencia. Los padres recuerdan la relación con su padre, relato que estuvo presente en todos los testimonios, independiente del quintil de ingresos:

"En su infancia nunca le faltó nada...su padre era preocupado de su familia hasta hoy...pero en cuanto a estar presente en cosas cotidianas como cambiar pañales...o... darles comida...eso...era responsabilidad de su mamá" (H10/9/4).

Los padres visualizan su experiencia en la infancia $\mathrm{y}$, por un lado, toman conciencia de la "difícil tarea que tuvieron que llevar a cabo sus propios padres” (H1/9/5). Así, se ponen en el lugar de ellos, generando sentimientos de agradecimiento, admiración y empatía con los desafíos y dificultades de ser padre.

\section{Categoría 2: Significado de la paternidad desde una perspectiva de género}

El ejercicio de la crianza es asignado al rol femenino, como parte de la conciencia colectiva de los participantes. Los nuevos padres relatan la forma en la que ellos han asumido parcialmente un rol, que siempre ha estado asociado a las mujeres y que optan por llevar a cabo de forma consciente. El cambio es limitado, porque aún hay necesidad de diferenciar a hombres de mujeres en cuanto a roles, ellos están dispuestos a asumir algunas actividades, principalmente las que les son placenteras, mientras que las mujeres asumen el resto, sin posibilidades de elección. El rol siempre presente en el discurso de todos los padres es el de proveedor de recursos, aunque más fuertemente en los quintiles bajos.

Todos los padres participantes buscan construir una relación activa con su hijo/a. Los hombres están dispuestos a jugar, a leerles cuentos, a pasar más tiempo con ellos, así como también poner las normas y hacer que sean cumplidas.

"Las actividades que hace con su hijo son las que disfruta... le gusta jugar, disfruta leerle un cuento y conversar con él; considera una suerte que se complementen con su mujer" (H11/11/5).
Otros aspectos relacionados con la crianza y cuidados de los niños/as están ausentes del discurso de padres y madres, cuando se trata de actividades que el hombre no está dispuesto a realizar. Actividades relacionadas con la alimentación, la higiene y el baño, hacer dormir a los niños/as, llevarlos a la supervisión de salud, entre otras, están fuera del discurso. Son actividades consideradas propias de la "biología femenina", incluso son motivo de mención cuando relatan las cosas que como padres modernos se han visto obligados a realizar y que les significó un sacrificio.

"...no cree en la igualdad de roles, porque hay roles determinados biológicamente, y en término de la relación con el niño, cuando es más pequeño es más la relación con la mamá. A medida que el niño crece...va...cambiando... aunque cree que su rol como soporte de la casa es básico” (H11/6/5).

\section{Categoría 3: Significado de la experiencia en la relación padre-hijo/a}

Los padres perciben inicialmente que son prescindibles del proceso de crianza de su hijo/a y que sus parejas no los dejan participar todo lo que quisieran.

"A partir del año, el padre comienza a sentir una mayor conexión con su hija, pues antes son pura mamá...lo que hace... difícil entrar en esa sintonía” (H1/11/5).

A medida que van interactuando más con el niño/a, sienten que van formando parte de su vida, estrechando y consolidando un vínculo mutuo. Tanto es así, que los padres se muestran interesados en usar el descanso posnatal parental. Lo anterior es una realidad para algunos de los padres de los quintiles más altos, quienes describieron la posibilidad de dedicar más tiempo al cuidado de sus hijos/as, permitiéndoseles realizar parte de su trabajo desde la casa.

"En su trabajo puede resguardar los tiempos para dedicarle a su hijo...me dejan trabajar medio tiempo desde la casa, puedo tomar permiso sin goce de sueldo cuando nacen los hijos... son súper abiertos a este tema" (H11/12/5)

Los padres manifestaron vivir el desarrollo de un vínculo afectivo con sus hijos/as, el que se desencadena a medida que el niño/a va creciendo y haciéndose más independiente. Dicho vínculo está propiciado fundamentalmente por iniciativa del niño/a.

"La relación afectiva es un proceso en constante crecimiento, aumenta en la medida que ella le estira los brazos, le da besos y con las palabras que expresa....es inmenso el afecto hacia su hija” (H2/10/5).

\section{Categoría 4: Significado de la experiencia de paternidad en la relación de pareja}

Los participantes coinciden en que el nacimiento de un hijo/a impacta en la relación de pareja, y que a su vez es la condición que los lleva a transformarse en 
familia. Al parecer influye positivamente cuando el padre participa de la crianza, involucrándose en diversas tareas.

"Percibe la transformación hacia la paternidad como positiva, porque han obtenido algo maravilloso, invaluable...ahora son familia...aunque tengan menos tiempo libre, fue una decisión tomada en pareja" (M1/25/5).

Existe un periodo de adaptación de la pareja frente a la llegada de un hijo/a, que los desafía a incorporar un nuevo actor en la dinámica que estaban acostumbrados a llevar. Durante este periodo, los padres consideran que pasan a segundo plano, porque toda la atención está en función del niño/a y sus necesidades.

"El ejercicio de la paternidad de su pareja ha influido mucho en su relación, porque ellos están juntos porque quieren, no están obligados y verlo como padre preocupado y queriendo a su hijo, compartiendo esta etapa, los hace quererse más, incluso cuando surgen problemas, la felicidad que sienten al estar juntos los hace sobreponerse a todo y los hace ser familia" (M9/13/4).

\section{Categoría 5: Ejercitando la paternidad y su relación con el sistema de salud}

La incorporación del padre en el sistema de salud surge en los relatos de los participantes, pareciendo estar determinada por el lugar que ocupan en la sociedad. En los quintiles de mayores ingresos, los padres perciben que participan, en la medida que tienen intención de hacerlo. Son capaces de darse cuenta de que los profesionales de la salud pueden tener o no la disposición a incorporarlos y se sienten con mayor poder para exigir sus derechos, en caso de que no los integren como quisieran.

"El sistema no lo apoyó en su proceso de paternidad, aunque... no considera que influya demasiado" (H2/18/5).

Por el contrario, las personas pertenecientes a los quintiles más bajos perciben escasas posibilidades de integración. Los padres coinciden en que participan como acompañante y no en su "rol de padre", el sistema de salud no da oportunidad de participar, aun cuando lo quieran.

"Los profesionales del servicio de urgencia no permitieron que el padre entrara a acompañar a su hija... incluso lo compararon con la madre, argumentando que tenía menos derechos respecto del cuidado de su hija" (M3/54/1).

Para ilustrar la experiencia del hombre al transformarse en padre por primera vez, se utilizó la figura de un árbol, donde el padre es como una semilla que tiene el potencial de germinar y con las categorías de género, relación de pareja, y desarrollo de un vínculo afectivo con su hijo/a, como sustratos, va configurando su paternidad, la que se ve determinada por el lugar que

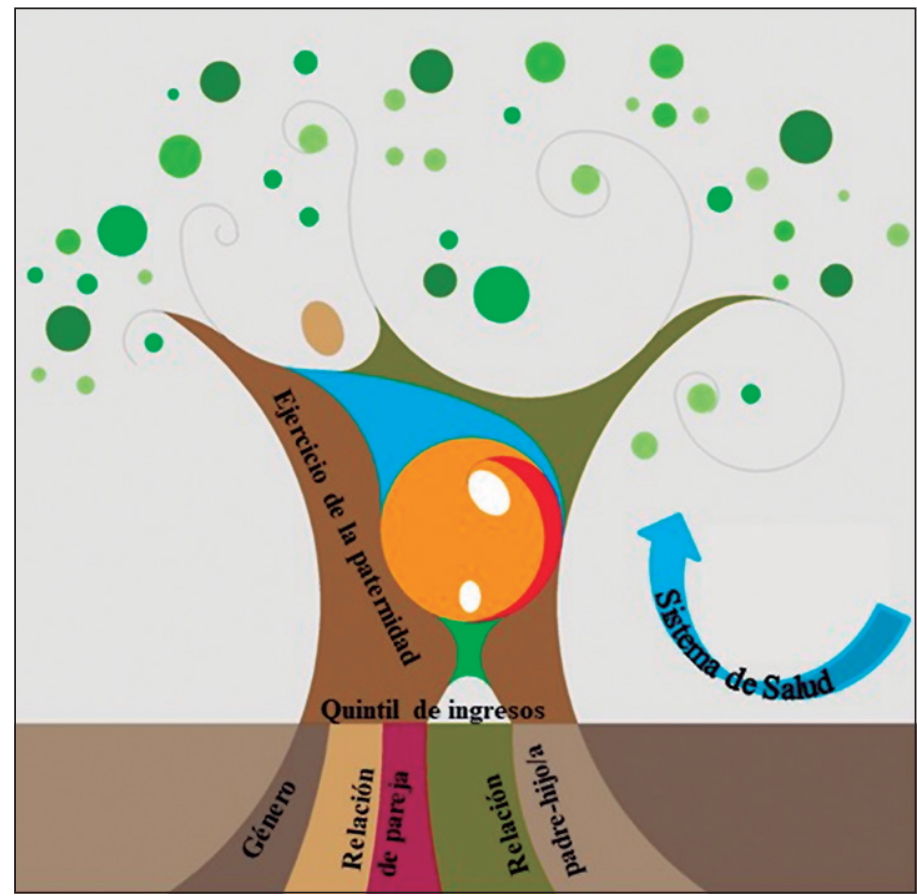

Figura 1. Significado y participación social del hombre al transformarse en padre.

ocupa en la sociedad. La familia, considerada como espacio social en donde ocurre la vivencia y el sistema de salud como condición externa, influyen fuertemente en las primeras oportunidades que tiene el hombre para ejercitar la paternidad en su contexto social (figura 1).

\section{Discusión}

La forma de vivir la paternidad es producto de la realidad social, determinada por diferencias existentes entre los grupos sociales ${ }^{18}$. Lo anterior, se articula con la vivencia única y personal por la que transitan los hombres en su proceso de transformación en padres, siendo productos de la historia personal y social, y de la forma en que la sociedad se organiza ${ }^{11}$.

En la vivencia de paternidad los hombres experimentan un amor profundo y la necesidad de priorizar la vida familiar. Coincidente con lo reportado en otros estudios $^{19-21}$, los participantes manifiestan tener expectativas e ilusión previo al parto, para luego sentirse sobrepasados por la realidad, la que difiere bastante de la imaginada, conflictuándolos 5 .

Los participantes buscan repetir la infancia que vivieron, cuando fue feliz y plena junto a sus padres, lo que coincide con otros estudios ${ }^{22}$. En contraste, cuando la experiencia con el propio padre no ha sido positiva, los participantes manifiestan la necesidad de no repetir patrones vividos en su infancia, surgiendo 
la necesidad de estar presente e involucrarse con su hijo/a en actividades cotidianas, lo que coincide con otros estudios ${ }^{21-25}$, demostrando que los padres de manera intuitiva, le asignan a este proceso la posibilidad de progresar y dar lo mejor de sí.

En el nuevo padre se manifiesta claramente una necesidad de cambio hacia otra visión con mayor participación en las actividades propias de la crianza. En las sociedades nórdicas, los hombres buscan estar involucrados, existiendo una legislación que lo promueve ${ }^{26}$.

En este estudio todos los padres manifiestan necesidad de involucrarse, independiente del quintil de ingreso, aunque la forma y medida en que se involucran sea diferente. La legislación chilena a pesar de que intenciona la participación activa de los padres en la crianza, no concreta posibilidades efectivas de incorporación ${ }^{4}$.

Los padres tienen necesidad de vivir su rol de manera diferente ${ }^{27}$. Buscan estar disponibles y presentes para sus hijos/as, y estudiar para acceder a una mejor calidad de vida para su familia ${ }^{27,28}$, como ocurre con los padres de los primeros quintiles de ingreso en este estudio.

Así, el ejercicio de la crianza está en proceso de cambio, aunque predomine el rol definido tradicionalmente como femenino, existiendo roles claramente demarcados. Lo anterior, coincide con lo reportado en otros estudios que señalan que existe delimitación en los roles asumidos e impuestos a hombres y mujeres, asociado a los círculos sociales a los que pertenecen $^{2,29,30}$.

Existe un cambio paulatino hacia un ejercicio más activo de la paternidad, principalmente en relación al juego, lo que coincide con otro estudio que destaca la participación del padre en actividades relacionadas con la entretención o con alta visibilidad pública ${ }^{31}$.

La consideración de actividades asociadas al cuidado del niño/a como parte de la "biología femenina", constituye un hallazgo de esta investigación, no se encontraron estudios que mencionaran este aspecto.

En este estudio los padres manifestaron sentirse prescindibles en el cuidado de sus hijos/as y señalaron la necesidad de que sus parejas les permitieran participar para ejercitar su rol. Lo anterior, coincide con otros estudios en que los padres manifestaban sentirse aislados y tristes por no poder interactuar más con sus hijos/as, debido a que parecen sólo necesitar de la madre ${ }^{32}$.

Los padres de este estudio relatan desarrollar una relación afectiva con su hijo/a. Otros estudios también han destacado que la estimulación del vínculo afecti$v^{33}$ entre padre e hijo/a, es fundamental para estimular el desarrollo del niño/a ${ }^{31,34}$, relevando la importancia de la participación del padre en la crianza ${ }^{12}$.

El proceso de desarrollo de un vínculo afectivo se desencadena en gran medida por iniciativa del niño/a, lo que no fue identificado en otras investigaciones que incorporan esta perspectiva ${ }^{25,35}$. Dado este hallazgo, es clave pensar intervenciones destinadas a estimular y promover la vinculación entre padres e hijos/as. Parece ser que el padre se mantiene pasivo a la interacción con el niño/a hasta que percibe la necesidad de contacto de él/ella. Se hace necesario apoyar a los nuevos padres en la búsqueda del conocimiento sensible de su hijo/a para promover su interacción precozmente.

En la relación de pareja se destaca que existe un periodo de adaptación frente a la llegada de un hijo/a, que desafía la dinámica que estaban acostumbrados a llevar. Coincidente con otros estudios, los padres consideran que pasan a segundo plano, porque la atención está en función del niño/a, lo que requiere de adaptación para lograr incorporar a los hombres, dándoles espacio para ejercer su rol ${ }^{20,35,36}$.

En consonancia con este estudio, se reporta que los nuevos padres están en búsqueda de un cambio del rol, esperando participar más activamente en las actividades cotidianas y no solo ejercer un rol de proveedor. Lo anterior contribuye a consolidar la relación de pareja, buscando el bienestar del grupo familiar ${ }^{5,35}$.

En cuanto a la acogida de los padres al sistema de salud, la literatura revisada no especifica cómo se incorporan, sino sugiere considerarlos en actividades relacionadas con el cuidado del niño/a ${ }^{5,10}$. En Chile se han establecido iniciativas para incorporar al padre como un acompañante de la madre ${ }^{4,37}$. Las potencialidades que surgen de este estudio realzan la posibilidad de considerar a la familia como protagonista del cuidado de los hijos/as, relevando a sus integrantes en el ejercicio parental, potenciándolos para un crecimiento y desarrollo integral de la familia.

Es interesante discutir cómo los contextos socioeconómicos determinan las prioridades al momento de ejercer el rol paterno. El desafío es ser sensible para visualizar e identificar la existencia de oportunidades para fomentar una vivencia plena de la paternidad en todos los contextos socioeconómicos.

Reconociendo que el proceso de transformarse en padre y madre tiene elementos comunes y especificidades necesarias de ser consideradas, se requiere apoyar especialmente a los padres para el ejercicio de la paternidad y desenvolvimiento familiar. También se hace necesario trabajar con las madres para apoyarlas en las formas en que pueden incentivar a sus parejas a participar.

Lo anterior, sugiere la necesidad de establecer políticas que fomenten el ejercicio de la paternidad considerando los diversos tipos de familia e incluir este conocimiento en la formación de profesionales de la salud.

Este estudio contribuye a la construcción de cono- 
cimiento respecto del ejercicio de la paternidad desde una perspectiva comprensiva. Para transferir sus resultados se requiere considerar las características específicas de este grupo y de las personas en las que podrían extrapolarse los resultados.

Este estudio no incluyó a familias del sector rural por lo que los resultados podrían no representar a este grupo. Se proyecta ampliar este estudio incluyendo a grupos que aporten mayor comprensión de la experiencia de paternidad.

\section{Responsabilidades Éticas}

Protección de personas y animales: Los autores declaran que los procedimientos seguidos se conformaron a las normas éticas del comité de experimentación humana responsable y de acuerdo con la Asociación Médica Mundial y la Declaración de Helsinki.

Confidencialidad de los datos: Los autores declaran que han seguido los protocolos de su centro de trabajo sobre la publicación de datos de pacientes.

\section{Derecho a la privacidad y consentimiento informa-} do: Los autores han obtenido el consentimiento informado de los pacientes y/o sujetos referidos en el artículo. Este documento obra en poder del autor de correspondencia.

\section{Conflicto de intereses}

Los autores declaran no tener conflicto de intereses.

\section{Financiamiento}

Este estudio fue desarrollado como parte de la tesis doctoral de la autora principal y se desarrolló con financiamiento propio de los autores.

\section{Referencias}

1. Márquez F, Bertolozzi MR. Rol Social y Participación del Padre en la Crianza de sus hijos/as en la Historia de Chile. Horiz Enfermería. 2013;24(1):42-9.

2. Valdés X. El lugar que habita el padre en Chile contemporáneo. Rev la Univ Boliv. 2009;8(23):385-410.

3. Cabieses B, Bernales M, Obach A, Pedrero $\mathrm{V}$. Una mirada a la vulnerabilidad social y sus consecuencias en la salud de las personas mayores en Chile. MINSAL, MINEDUC, UDD, OPS, OMS, América Solidaria. 2016. 1-484 p.

4. Atalah SE, Cordero VM, Guerra Z ME. Monitoreo de la implementación del programa Chile Crece Contigo en el embarazo: 2008-2011. Rev Chil pediatría. 2013;78(4):310-6.

5. Poh HL, Koh SS, Seow HC, He HG First-time fathers' experiences and needs during pregnancy and childbirth: a descriptive qualitative study. Midwifery. 2014;30(6):779-87.

6. Reimer T. Measuring German Fathers' Involvement in Childcare. Men Masc. 2017;20(5):588-608

7. Fenwick J, Bayes S, Johansson M. A qualitative investigation into the pregnancy experiences and childbirth expectations of Australian fathers-tobe. Sex Reprod Healthc [Internet]. 2012;3(1):3-9. Available from: http:// dx.doi.org/10.1016/j.srhc.2011.11.001.

8. Márquez F, Lucchini C, Bertolozzi MR, Bustamante C, Strain H, Alcayaga $\mathrm{C}$, et al. Being a first-time father.
Their experiences and meanings : A Qualitative Systematic Review. Rev Chil Pediatr [Internet]. 2019;90(1):7887. Available from: https://www. revistachilenadepediatria.cl/index.php/ rchped/article/view/821/935.

9. Johansson T. Fatherhood in Transition: Paternity Leave and Changing Masculinities. J Fam Commun [Internet]. 2011;11(3):165-80. Available from: http:// www.tandfonline.com/doi/abs/10.1080 /15267431.2011.561137 [citado el 11 de agosto de 2013].

10. Chin R, Daiches A, Hall P. A qualitative exploration of first-time fathers ' experiences of becoming a father. 2011;84(7):19-24.

11. Egry E, Hino P. Las necesidades en salud en la perspectiva de la Atención Básica. Guía para investigadores. $1^{\mathrm{a}}$ ed. Sao Paulo: Escuela de Enfermeria de la USP; 2009. 1-120 p.

12. MIDEPLAN. Evaluación y Monitoreo Sistema Integral de Protección a la Infancia Chile Crece Contigo. 2011.

13. Bedregal P, Torres A. Chile Crece Contigo: el desafío de crear políticas públicas intersectoriales. Inst Políticas Públicas. 2013;1(19):1-8.

14. Bedregal P, Torres A, Carvallo C. Chile Crece Contigo: el Desafío de la Protección Social a la Infancia . 2014.

15. Minayo MC de S. Los conceptos estructurantes de la investigación cualitativa. Salud Colect. 2010;6(3):25161.

16. Tong A, Sainsbury P, Craig J. Consolidated criteria for reporting qualitative research (COREQ): a 32-item checklist for interviews and focus groups. Int J Qual Heal Care. 2007;19(6):349-57.

17. Streubert H, Carpenter D. Qualitative Research in Nursing: Advancing the Humanistic Imperative. $5^{\mathrm{a}}$ ed. Lippincott Williams \& Wilkins; 2010. 1-496 p.

18. Morales-Borrero C, Borde E. ¿Determinación social o determinantes sociales? Diferencias conceptuales e implicaciones praxiológicas. Rev salud pública. 2013;15(6):797-808.

19. Teixeira RC, Mandú ENT, Corrêa ÁC de $\mathrm{P}$, Marcon SS. Vivências e necessidades de saúde de homens no período pósnascimento de um filho. Rev Bras Enferm [Internet]. 2014;67(5):780-7. Available from: http://www.scielo.br/ scielo.php?script $=$ sci_arttext\&pid $=$ S0034$71672014000500780 \& \operatorname{lng}=$ pt\&nrm $=$ iso $\& \mathrm{t}$ $\operatorname{lng}=\mathrm{en}$

20. Cubbins LA, Sepavich D, Killpack S, Hill CV. Assessing Determinants of Father's Involvement with His Partner's Pregnancy and His Child's Well-being: The Father Resources Survey Instrument. Men Masc. 2018;21(1):3-34.

21. Coltart C, Henwood K. On paternal subjectivity: a qualitative longitudinal and psychosocial case analysis of men's classed positions and transitions to first-time fatherhood. Qual Res. 2012;12(1):35-52.

22. Krob A, Piccinini C, Silva M. A transicao para a paternidade: Da Gestacao ao Segundo mes de vida do bebe. Psicol USP. 2009;20(2):269-91.

23. Gonçalves TR, Eguia L, Silva R, Cássia 
R De, Lopes S, Augusto C. Experiência da Paternidade aos Três Meses do Bebê. Psicol Reflexão e Crítica. 2009;26(3):599608.

24. Hamilton A, de Jonge D. The impact of becoming a father on other roles: An ethnographic study. J Occup Sci [Internet]. 2010;17(1):40-6. Available from: http://search.ebscohost.com/login. aspx?direct $=$ true $\& \mathrm{db}=$ psyh \&AN $=2010$ 06954-006\&lang=es\&site=ehost-live

25. Gabriel MR, Dias ACG. Percepções sobre a paternidade: descrevendo a si mesmo e o próprio pai como pai. Estud Psicol. 2011;16(3):253-61.

26. Eerola JP, Huttunen J. Metanarrative of the "New Father" and Narratives of Young Finnish First-Time Fathers. Father A J Theory, Res Pract about Men as Father [Internet]. 2011;9(3):211-31. Available from: http://mensstudies.metapress. com/openurl.asp?genre $=$ article $\&$ id $=\mathrm{d}$ oi:10.3149/fth.0903.211 [citado el 24 de octubre de 2014].

27. Barclay L, Lupton D. The experiences of new fatherhood: a socio-cultural analysis.
J Adv Nurs [Internet]. 1999;29(4):101320. Available from: http://www.ncbi.nlm. nih.gov/pubmed/10215995.

28. Berton F, Bureau MC, Rist B. Father Figures and Cultural Diversity in France. Men Masc. 2017;20(5):552-69.

29. Jadue G. Transformaciones familiares en Chile: riesgo creciente para el desarrollo emocional, psicosocial y la educacion de los hijos. Estud pedagógicos. 2003;29:11526.

30. Hipp TR. Orígenes del matrimonio y de la familia modernos. Rev Austral Ciencias Soc [Internet]. 2006;11:59-78. Available from: http://mingaonline.uach.cl/scielo. php?script=sci_arttext\&pid=S0718$17952006000200004 \& \operatorname{lng}=\mathrm{es} \& \mathrm{nrm}=\mathrm{iso} \& \mathrm{t}$ lng=es

31. Rehel EM. When dad stays home too: Paternity Leave, Gender, and Parenting. Gend Soc. 2014;28(1):110-32.

32. Nyström K, Öhrling K. Parenthood experiences during the child' s first year: literature review. J Adv Nurs. 2004;46(3):319-30.

33. Bowlby J. El apego y la pérdida. $3^{\mathrm{a}} \mathrm{ed}$.
Barcelona: Paidós Ibérica; 1998. 524 p.

34. Hall RAS, De Waard IEM, Tooten A, Hoffenkamp HN, Vingerhoets AJJM, van Bakel HJA. From the father's point of view: How father's representations of the infant impact on father-infant interaction and infant development. Early Hum Dev [Internet]. 2014;90(12):877-83. Available from: http://dx.doi.org/10.1016/j. earlhumdev.2014.09.010.

35. Iwata $\mathrm{H}$. Experiences of Japanese men during the transition to fatherhood. $\mathrm{J}$ Transcult Nurs [Internet]. 2014;25(2):159-66. Available from: http://www.ncbi.nlm.nih.gov/ pubmed/24518060.

36. Brandth B, Kvande E. Masculinity and Fathering Alone during Parental Leave. Men Masc. 2018;21(1):72-90.

37. Ministerio de salud. Programa nacional de salud de la infancia con enfoque integral [Internet]. 2013. 184 p. Available from: http://web.minsal.cl/sites/default/files/ files/2013_Programa Nacional de Salud de la infancia con enfoque integral.pdf. 